\title{
Amount Recovered Normalized by Dose
}

National Cancer Institute

\section{Source}

National Cancer Institute. Amount Recovered Normalized by Dose. NCI Thesaurus. Code C92364.

The cumulative amount recovered from the specimen type, from dosing to end of the current collection interval divided by the dose. 